International Journal of Pure and Applied Mathematics

Volume $91 \quad$ No. 1 2014, 87-101

ISSN: 1311-8080 (printed version); ISSN: 1314-3395 (on-line version)

url: http://www.ijpam.eu

doi: http://dx.doi.org/10.12732/ijpam.v91i1.10

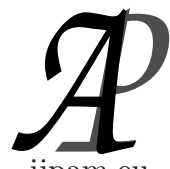

ijpam.eu

\title{
NIL 3-ARMENDARIZ RINGS RELATIVE TO A MONOID
}

\author{
Eltiyeb Ali ${ }^{1 \S}$, Ayoub Elshokry ${ }^{2}$ \\ ${ }^{1,2}$ Department of Mathematics \\ Northwest Normal University \\ Lanzhou, 730070, P.R. CHINA \\ ${ }^{1,2}$ Department of Mathematics \\ University of Khartoum \\ Omdurman, SUDAN
}

\begin{abstract}
For a monoid $M$, we introduce nil 3- $M$-Armendariz, which are a common generalization of nil 3 -Armendariz and $3-M$-Armendariz rings, and investigates their properties. We show that a ring $R$ is nil $3-M$-Armendariz ring if and only if for any $n \in \mathbb{N}, T_{n}(R)$ is nil 3-M-Armendariz, where $M$ is a monoid. Also we show that if a ring $R$ is semicommutative which is also nil 3 - $M$-Armendariz, then $R$ is nil 3- $(M \times N)$-Armendariz, where $N$ is a unique product monoid.
\end{abstract}

AMS Subject Classification: 16S36, 16U20, 16N60, 16U99

Key Words: unique product monoid, 3-Armendariz ring, nil 3-Armendariz ring, 3- $M$-Armendariz ring, nil 3- $M$-Armendariz

\section{Introduction}

Throughout this article, $R$ and $M$ denote an associative ring, not necessary with identity and a monoid, respectively. Given a ring $R$, the polynomial ring over $R$ is denoted by $R[x]$. Rege and Chhawchharia [12], introduced the notion of an Armendariz ring. A ring $R$ is called Armendariz if whenever polynomials $f(x)=$

Received: November 30, 2013

(C) 2014 Academic Publications, Ltd.

${ }^{\S}$ Correspondence author url: www.acadpubl.eu 
$a_{0}+a_{1} x+\cdots+a_{n} x^{n}, g(x)=b_{0}+b_{1} x+\cdots+b_{m} x^{m} \in R[x]$, satisfy $f(x) g(x)=0$, then $a_{i} b_{j}=0$ for each $i$ and $j$. The name "Armendariz ring" was chosen because Armendariz [8, Lemma 1], has shown that a reduced ring (i.e., a ring without nonzero nilpotent elements) satisfies this condition. Some properties of Armendariz rings have been studied in Hong and et al. [3], Anderson and Camillo [4], Kim and Lee [15], Huh et al. [2] and Lee and Wong [17]. In [20], Suiyi, introduced the notion of 3-Armendariz rings. A ring $R$ is called a 3Armendariz ring if whenever polynomials $f(x)=a_{0}+a_{1} x+\cdots+a_{n} x^{n}, g(x)=$ $b_{0}+b_{1} x+\cdots+b_{m} x^{m}, h(x)=c_{0}+c_{1} x+\cdots+c_{r} x^{r} \in R[x]$, satisfy $f(x) g(x) h(x)=0$, then $a_{i} b_{j} c_{k}=0$, for all $i, j$ and $k$. Armendariz rings are thus a generalization of reduced rings, and therefore, nilpotent elements play an important role in this class of rings. In fact, in [4], Anderson and Camillo proved that if $n \geq 2$, then $R[x] /\left(x^{n}\right)$ is an Armendariz ring if and only if $R$ is reduced. Zhongkui [10], studied a generalization of Armendariz rings, which are called $M$-Armendariz rings, where $M$ is a monoid. A ring $R$ is called $M$-Armendariz if whenever elements $\alpha=a_{1} g_{1}+\cdots+a_{n} g_{n}, \beta=b_{1} h_{1}+\cdots+b_{m} h_{m} \in R[M]$, satisfy $\alpha \beta=0$, then $a_{i} b_{j}=0$ for each $i, j$, where $g_{i}, h_{j} \in M$. Elshokry and et al. [1], studied a generalization of $M$-Armendariz rings, which are called 3 - $M$-Armendariz rings, where $M$ is a monoid. A ring $R$ is called 3-M-Armendariz if whenever elements $\alpha=a_{1} g_{1}+\cdots+a_{n} g_{n}, \beta=b_{1} h_{1}+\cdots+b_{m} h_{m}, \gamma=c_{1} l_{1}+\cdots+c_{r} l_{r} \in R[M]$, satisfy $\alpha \beta \gamma=0$, then $a_{i} b_{j} c_{k}=0$ for each $i, j, k$, where $g_{i}, h_{j}, l_{k} \in M$. Armendariz ring are abelian by Kim and Lee [15]. Subrings of $M$-Armendariz rings are also $M$-Armendariz by Zhongkui [10]. Subrings of 3-Armendariz rings are also 3 -Armendariz by Suiyi [20]. Subrings of $3-M$-Armendariz rings are also 3$M$-Armendariz by Elshokry and et al. [1]. According to Antoine [16]. A ring $R$ is called nil-Armendariz if whenever two polynomials $f(x), g(x) \in R[x]$, satisfy $f(x) g(x) \in \operatorname{nil}(R)[x]$ then $a b \in \operatorname{nil}(R)$ for all $a \in \operatorname{coe} f(f(x))$ and $b \in$ coef $(g(x))$, where coef $(f(x))$ denote the subset of $R$ of the coefficients of $f(x)$. Mohammed and et al. [14], introduced the notion of nil $M$-Armendariz rings. A ring $R$ is said to be nil $M$-Armendariz rings, if whenever elements $\alpha=a_{1} g_{1}+$ $\cdots+a_{n} g_{n}, \beta=b_{1} h_{1}+\cdots+b_{m} h_{m} \in R[M]$, satisfy $\alpha \beta \in \operatorname{nil}(R)[M]$, when $a_{i} b_{j} \in$ $\operatorname{nil}(R)$ for each $i, j$. Zhang Cuiping and Chen Jianlong [21], introduced the notion of weak $M$-Armendariz rings. A ring $\mathrm{R}$ is said to be weak M-Armendariz if whenever elements $\alpha=a_{1} g_{1}+\cdots+a_{n} g_{n}$ and $\beta=b_{1} h_{1}+\cdots+b_{m} h_{m} \in R[M]$, satisfy $\alpha \beta=0$, then $a_{i} b_{j} \in \operatorname{nil}(R)$ for each $i, j$. In [11], Liu and Zhao introduced weak Armendariz rings which a generalization of Armendariz rings. A ring $R$ is called weak Armendariz if whenever the product of two polynomials is zero then the product of their coefficients is nilpotent. Weak Armendariz rings have the property that motivates the study of the nilpotent elements in this class of 
rings. Recall that a ring $R$ is called semicommutative if for all $a, b \in R, a b=0$ implies $a R b=0$. Yang Suiyi [20], introduced the notion of Condition $(P)$, for all $a, b, c \in R$, if $(a b c)^{2}=0$, then $a b c=0$. In [19], Wu Hui-feng introduced the concept of weak 3-Armendariz ring which a generalization of 3-Armendariz ring and weak Armendariz ring and investigate their properties. A ring $R$ is called weak 3-Armendariz if whenever the product of three polynomials is zero then the product of their coefficients is nilpotent. If $M=\{e\}$, then every ring is 3 - $M$-Armendariz, so it is nil $3-M$-Armendariz. Thus nil $3-M$-Armendariz rings need not be nil 3 -Armendariz. Hence nil 3 - $M$-Armendariz rings are a common generalization of $3-M$-Armendariz rings and nil 3-Armendariz rings. If $M=(\mathbb{N} \cup\{0\},+)$, then a ring $R$ is nil 3 - $M$-Armendariz if and only if $R$ is nil-3-Armendariz.

Recall that a monoid $M$ is called a $u . p .-$ monoid (unique product monoid) if for any two nonempty finite subsets $A, B \subseteq M$ there exists an element $g \in M$ uniquely presented in the form $a b$ where $a \in A$ and $b \in B$. The class of u.p.monoids is quite large and important (see Birkenmeier and Park [9], Passman [5]). For example, this class includes the right or left ordered monoids, submonoids of a free group, and torsion-free nilpotent groups. Every u.p.-monoid $M$ has non unity element of finite order. Motivated by results in Elshokry and et al. [1], Suiyi [20], Zhongkui [10], Habibi and Moussavi [13], Antoine [16], Ebrahim Hashemi [7] and Mohammed and et al. [14], we will investigate a generalization of $3-M$-Armendariz which are called nil $3-M$-Armendariz. For a ring $R$, we denote by $R[M]$ the monoid ring over $R$, and by $\operatorname{nil}(R)$ the set of nilpotent elements in $R$. If $\alpha \in R[M]$, coef $(\alpha)$ denote the subset of $R$ of the coefficients of $\alpha$.

\section{Nil 3-Armendariz Rings Relative to a Monoid}

For a monid $M$, e will always stand for the identity of $M$. We denote by $T_{n}(R)$ the $n \times n$ upper triangular matrix over a ring $R$.

Definition 2.1. $\quad$ [6, Definition 2.5]. A ring $R$ is said to be nil 3-Armendariz if whenever polynomials $f(x), g(x), h(x) \in R[x]$, satisfy $f(x) g(x) h(x) \in \operatorname{nil}(R)[x]$ then $a b c \in \operatorname{nil}(R)$ for all $a \in \operatorname{coe} f(f(x)), b \in \operatorname{coe} f(g(x))$ and $c \in \operatorname{coe} f(h(x))$.

Definition 2.2. Let $M$ be a monoid. A ring $R$ is called nil 3-Armendariz relative to $M$ (nil 3 - $M$-Armendariz) if whenever elements $\alpha=a_{1} g_{1}+\cdots+$ $a_{n} g_{n}, \beta=b_{1} h_{1}+\cdots+b_{m} h_{m}$ and $\gamma=c_{1} l_{1}+\cdots+c_{r} l_{r} \in R[M]$, satisfy $\alpha \beta \gamma \in$ $\operatorname{nil}(R)[M]$, then $a_{i} b_{j} c_{k} \in \operatorname{nil}(R)$ for each $i, j, k$. 
Lemma 2.3. [18, Proposition 1]. If $R$ a reduced rings then $R$ satisfies condition $(P)$, but the converse is not true.

Clearly, any subrings of nil 3- $M$-Armendariz rings are nil 3- $M$-Armendariz and any $3-M$-Armendariz ring is nil $3-M$-Armendariz. In the following, we will see that the converse is not true.

Proposition 2.4. Let $R$ be a ring and $M$ a monoid. Then $R$ is a nil $3-M$-Armendariz ring if and only if, for any $n, T_{n}(R)$ is a nil $3-M$-Armendariz ring.

Proof. We note that any subring of nil 3 - $M$-Armendariz ring is a nil 3 $M$-Armendariz ring. Thus if $T_{n}(R)$ is a nil $3-M$-Armendariz ring, then so is $R$. Conversely, let $\alpha=A_{1} g_{1}+\cdots+A_{n} g_{n}, \beta=B_{1} h_{1}+\cdots+B_{m} h_{m}$ and $\gamma=C_{1} l_{1}+\cdots+C_{r} l_{r}$ be elements of $T_{n}(R)[M]$. It is easy to see that there exists an isomorphism of rings $T_{n}(R)[M] \rightarrow T_{n}(R[M])$ define by:

$$
\begin{aligned}
& \sum_{i=1}^{p}\left(\begin{array}{ccccc}
a_{11}^{i} & a_{12}^{i} & a_{13}^{i} & \cdots & a_{1 n}^{i} \\
0 & a_{22}^{i} & a_{23}^{i} & \cdots & a_{2 n}^{i} \\
0 & 0 & a_{33}^{i} & \cdots & a_{3 n}^{i} \\
\vdots & \vdots & \vdots & \ddots & \vdots \\
0 & 0 & 0 & \cdots & a_{n n}^{i}
\end{array}\right) g_{i} \mapsto \\
&\left(\begin{array}{cccccc}
\sum_{i=1}^{p} a_{11}^{i} g_{i} & \sum_{i=1}^{p} a_{12}^{i} g_{i} & \sum_{i=1}^{p} a_{13}^{i} g_{i} & \cdots & \sum_{i=1}^{p} a_{1 n}^{i} g_{i} \\
0 & \sum_{i=1}^{p} a_{22}^{i} g_{i} & \sum_{i=1}^{p} a_{23}^{i} g_{i} & \cdots & \sum_{i=1}^{p} a_{2 n}^{i} g_{i} \\
0 & 0 & \sum_{i=1}^{p} a_{33}^{i} g_{i} & \cdots & \sum_{i=1}^{p} a_{3 n}^{i} g_{i} \\
\vdots & \vdots & \vdots & \ddots & \\
0 & 0 & 0 & \cdots & \sum_{i=1}^{p} a_{n n}^{i} g_{i}
\end{array}\right)
\end{aligned}
$$

Assume that $\alpha \beta \gamma \in \operatorname{nil}\left(T_{n}(R)\right)[M]$. Let

$$
A_{i}=\left(\begin{array}{ccccc}
a_{11}^{i} & a_{12}^{i} & a_{13}^{i} & \cdots & a_{1 n}^{i} \\
0 & a_{22}^{i} & a_{23}^{i} & \cdots & a_{2 n}^{i} \\
0 & 0 & a_{33}^{i} & \cdots & a_{3 n}^{i} \\
\vdots & \vdots & \vdots & \ddots & \vdots \\
0 & 0 & 0 & \cdots & a_{n n}^{i}
\end{array}\right), B_{j}=\left(\begin{array}{ccccc}
b_{11}^{j} & b_{12}^{j} & b_{13}^{j} & \cdots & b_{1 n}^{j} \\
0 & b_{22}^{j} & b_{23}^{j} & \cdots & b_{2 n}^{j} \\
0 & 0 & b_{33}^{j} & \cdots & b_{3 n}^{j} \\
\vdots & \vdots & \vdots & \ddots & \vdots \\
0 & 0 & 0 & \cdots & b_{n n}^{j}
\end{array}\right)
$$


and

$$
C_{k}=\left(\begin{array}{ccccc}
c_{11}^{k} & c_{12}^{k} & c_{13}^{k} & \cdots & c_{1 n}^{k} \\
0 & c_{22}^{k} & c_{23}^{k} & \cdots & c_{2 n}^{k} \\
0 & 0 & c_{33}^{k} & \cdots & c_{3 n}^{k} \\
\vdots & \vdots & \vdots & \ddots & \vdots \\
0 & 0 & 0 & \cdots & c_{n n}^{k}
\end{array}\right)
$$

Then we have

$$
\begin{aligned}
& \left(\begin{array}{ccccc}
\sum_{i=1}^{p} a_{11}^{i} g_{i} & \sum_{i=1}^{p} a_{12}^{i} g_{i} & \sum_{i=1}^{p} a_{13}^{i} g_{i} & \cdots & \sum_{i=1}^{p} a_{1 n}^{i} g_{i} \\
0 & \sum_{i=1}^{p} a_{22}^{i} g_{i} & \sum_{i=1}^{p} a_{23}^{i} g_{i} & \cdots & \sum_{i=1}^{p} a_{2 n}^{i} g_{i} \\
0 & 0 & \sum_{i=1}^{p} a_{33}^{i} g_{i} & \cdots & \sum_{i=1}^{p} a_{3 n}^{i} g_{i} \\
\vdots & \vdots & \vdots & \ddots & \vdots \\
0 & 0 & 0 & \cdots & \sum_{i=1}^{p} a_{n n}^{i} g_{i}
\end{array}\right) \\
& \times\left(\begin{array}{ccccc}
\sum_{j=1}^{q} b_{11}^{j} h_{j} & \sum_{j=1}^{q} b_{12}^{j} h_{j} & \sum_{j=1}^{q} b_{13}^{j} h_{j} & \cdots & \sum_{j=1}^{q} b_{1 n}^{j} h_{j} \\
0 & \sum_{j=1}^{q} b_{22}^{j} h_{j} & \sum_{j=1}^{q} b_{23}^{j} h_{j} & \cdots & \sum_{j=1}^{q} b_{2 n}^{j} h_{j} \\
0 & 0 & \sum_{j=1}^{q} b_{33}^{j} h_{j} & \cdots & \sum_{j=1}^{q} b_{3 n}^{j} h_{j} \\
\vdots & \vdots & \vdots & \ddots & \vdots \\
0 & 0 & 0 & \cdots & \sum_{j=1}^{q} b_{n n}^{j} h_{j}
\end{array}\right) \\
& \times\left(\begin{array}{ccccc}
\sum_{k=1}^{d} c_{11}^{k} l_{k} & \sum_{k=1}^{d} c_{12}^{k} l_{k} & \sum_{k=1}^{d} c_{13}^{k} l_{k} & \cdots & \sum_{k=1}^{d} c_{1 n}^{k} l_{k} \\
0 & \sum_{k=1}^{d} c_{22}^{k} l_{k} & \sum_{k=1}^{d} c_{23}^{k} l_{k} & \cdots & \sum_{k=1}^{d} c_{2 n}^{k} l_{k} \\
0 & 0 & \sum_{k=1}^{d} c_{33}^{k} l_{k} & \cdots & \sum_{k=1}^{d} c_{3 n}^{k} l_{k} \\
\vdots & \vdots & \vdots & \ddots & \vdots \\
0 & 0 & 0 & \cdots & \sum_{k=1}^{d} c_{n n}^{k} l_{k}
\end{array}\right) \in \operatorname{nil}\left(T_{n}(R[M])\right) .
\end{aligned}
$$

Because $T_{n}(R)[M] \cong T_{n}(R[M])$. Also

$$
\operatorname{nil}\left(T_{n}(R)\right)=\left(\begin{array}{cccc}
\operatorname{nil}(R) & R & R & R \\
0 & \operatorname{nil}(R) & R & R \\
\vdots & \vdots & \ddots & \vdots \\
0 & 0 & \cdots & \operatorname{nil}(R)
\end{array}\right) .
$$

It follows that

$$
\left(\sum_{i=1}^{p} a_{s s}^{i} g_{i}\right)\left(\sum_{j=1}^{q} b_{s s}^{j} h_{j}\right)\left(\sum_{k=1}^{d} c_{s s}^{k} l_{k}\right) \in \operatorname{nil}(R)[M],
$$

for $s=1,2, \cdots, n$. 
Since $R$ is nil 3-M-Armendariz, there exists $m_{i j k s} \in \mathbb{N}$ such that

$$
\left(a_{s s}^{i} b_{s s}^{j} c_{s s}^{k}\right)^{m_{i j k s}}=0
$$

for any $s$ and any $i, j, k$. Let $m_{i j k}=\max \left\{m_{i j k 1}, m_{i j k 2}, \cdots, m_{i j k n}\right\}$. Then

$$
\begin{aligned}
& \left(A_{i} B_{j} C_{k}\right)^{m_{i j k}}=\left(\begin{array}{cccc}
\left(a_{11}^{i} b_{11}^{j} c_{11}^{k}\right)^{m_{i j k}} & * & \cdots & * \\
0 & \left(a_{22}^{i} b_{22}^{j} c_{22}^{k}\right)^{m_{i j k}} & \cdots & * \\
\vdots & \vdots & \ddots & * \\
0 & 0 & \cdots & \left(a_{n n}^{i} b_{n n}^{j} c_{n n}^{k}\right)^{m_{i j k}}
\end{array}\right) \\
& =\left(\begin{array}{cccc}
0 & * & \cdots & * \\
0 & 0 & \cdots & * \\
\vdots & \vdots & \ddots & \vdots \\
0 & 0 & \cdots & 0
\end{array}\right) .
\end{aligned}
$$

Thus $\left(\left(A_{i} B_{j} C_{k}\right)^{m_{i j k}}\right)^{n}=0$ and so $A_{i} B_{j} C_{k} \in \operatorname{nil}\left(T_{n}(R)\right)$, for each $i, j, k$. This shows that $T_{n}(R)$ is nil 3 - $M$-Armendariz ring.

Corollary 2.5. Let $M$ be a monoid. If a ring $R$ is $3-M$-Armendariz, then, for any $n, T_{n}(R)$ is nil 3-M-Armendariz.

Now we can give the example of nil 3 - $M$-Armendariz ring which is not 3-M-Armendariz.

Example 2.6. Let $M$ be a monoid. Let $S$ be a nil 3 - $M$-Armendariz ring. Then the ring

$$
R_{n}=\left\{\left(\begin{array}{ccccc}
a & a_{12} & a_{13} & \cdots & a_{1 n} \\
0 & a & a_{23} & \cdots & a_{2 n} \\
0 & 0 & a & \cdots & a_{3 n} \\
\vdots & \vdots & \vdots & \ddots & \vdots \\
0 & 0 & 0 & \cdots & a
\end{array}\right) \mid a, a_{i j} \in S\right\}
$$

is not 3 - $M$-Armendariz by Elshokry and et al. [1, Example 2.14] when $n \geq 4$, but $R_{n}$ is nil 3 - $M$-Armendariz by Proposition 2.4, since any subring of nil 3 - $M$-Armendariz ring is nil 3 - $M$-Armendariz.

From Proposition 2.4, one may suspect that if $R$ is nil 3 - $M$-Armendariz then every n-by-n full matrix ring $M_{n}(R)$ over $R$ is nil 3-M-Armendariz, where $n \geq 2$. But the following example erases the possibility. 
Example 2.7. Let $M$ be a monoid with $|M| \geq 2$ and $R$ a ring with identity. Take $e \neq g \in M$. Let $S=M_{2}(F)$. Let

$$
\begin{gathered}
\alpha=\left(\begin{array}{ll}
0 & 1 \\
0 & 0
\end{array}\right) e+\left(\begin{array}{ll}
1 & 0 \\
0 & 0
\end{array}\right) g, \beta=\left(\begin{array}{ll}
1 & 0 \\
0 & 1
\end{array}\right) g, \\
\gamma=\left(\begin{array}{ll}
1 & 1 \\
0 & 0
\end{array}\right) e+\left(\begin{array}{cc}
0 & 0 \\
-1 & -1
\end{array}\right) g
\end{gathered}
$$

be elements in $S[M]$. Then $\alpha \beta \gamma=0$. But

$$
\left(\begin{array}{ll}
1 & 0 \\
0 & 0
\end{array}\right)\left(\begin{array}{ll}
1 & 0 \\
0 & 1
\end{array}\right)\left(\begin{array}{ll}
1 & 1 \\
0 & 0
\end{array}\right)=\left(\begin{array}{ll}
1 & 1 \\
0 & 0
\end{array}\right)
$$

is not nilpotent. Thus $S$ is not nil $3-M$-Armendariz.

Corollary 2.8. Let $M$ be a monoid and $R$ a ring. Then $R$ is nil 3-MArmendariz, If and only if the trivial extension $T(R, R)=\left\{\left(\begin{array}{cc}a & b \\ 0 & a\end{array}\right) \mid a, b \in R\right\}$ is a nil 3-M-Armendariz.

Proof. It follows from Proposition 2.4.

Proposition 2.9. The class of nil 3-M-Armendariz rings is closed under finite direct products.

Proof. Let $R=\prod_{s \in \beta} R_{s}$ be the finite direct product of $R_{s}$ where $\beta=$ $\{1,2, \cdots, p\}, R_{s}$ is nil 3-M-Armendariz. Suppose $\alpha \beta \gamma \in \operatorname{nil}(R)[M]$ for some elements $\alpha=a_{1} g_{1}+a_{2} g_{2}+\cdots+a_{n} g_{n}, \beta=b_{1} h_{1}+b_{2} h_{2}+\cdots+b_{m} h_{m}$ and $\gamma=c_{1} l_{1}+$ $c_{2} l_{2}+\cdots+c_{r} l_{r} \in R[M]$, where $a_{i}=\left(a_{i 1}, a_{i 2}, \cdots, a_{i p}\right), b_{j}=\left(b_{j 1}, b_{j 2}, \cdots, b_{j p}\right), c_{k}=$ $\left(c_{k 1}, c_{k 2}, \cdots, c_{k p}\right)$, are elements of the product ring $R$. Set $\alpha_{s}=\sum_{i=1}^{n} a_{i s} g_{i}$, $\beta_{s}=\Sigma_{j=1}^{m} b_{j s} h_{j}$ and $\gamma_{s}=\Sigma_{k=1}^{r} c_{k s} l_{k} \in R[M]$. Since $\alpha \beta \gamma \in \operatorname{nil}(R)[M]$ then $\Sigma_{i+j+k=u} a_{i} b_{j} c_{k} \in \operatorname{nil}(R), 1 \leq u \leq n+m+r$. So $\Sigma_{i+j+k=u}\left(a_{i 1} b_{j 1} c_{k 1}, \cdots, a_{i p} b_{j p} c_{k p}\right)=$ 0 , and so $\Sigma_{i+j+k=u}\left(a_{i s} b_{j s} c_{k s}\right) \in \operatorname{nil}(R), 1 \leq s \leq p$. Thus $\alpha_{s} \beta_{s} \gamma_{s} \in \operatorname{nil}\left(R_{s}\right)[M], 1 \leq$ $s \leq p$. Since $R_{s}$ is nil 3-M-Armendariz, then we have $a_{i s} b_{j s} c_{k s} \in \operatorname{nil}\left(R_{s}\right)$. Now, for each $i, j, k$, there exist positive integers $m_{i j k s}$ such that $\left(a_{i s} b_{j s} c_{k s}\right)^{m_{i j k s}}=0$, in the ring $R_{s}, 1 \leq s \leq p$. If we take $m_{i j k}=\max \left\{m_{i j k s}: 1 \leq s \leq p\right\}$, then it is clear that $\left(a_{i s} b_{j s} c_{k s}\right)^{m_{i j k}}=0$. Therefore $a_{i} b_{j} c_{k} \in \operatorname{nil}(R)$. This means that $R$ is nil 3-M-Armendariz.

Theorem 2.10. Let $M$ be a u.p.-monoid and nil $(R)$ an ideal of $R$. Then $R$ is nil 3-M-Armendariz. 
Proof. Let $\alpha=\sum_{i=1}^{n} a_{i} g_{i}, \beta=\sum_{j=1}^{m} b_{j} h_{j}$ and $\gamma=\sum_{k=1}^{r} c_{k} l_{k}$ in $R[M]$, satisfy $\alpha \beta \gamma \in \operatorname{nil}(R)[M]$. Since $\operatorname{nil}(R)$ is an ideal of $R$, the $\operatorname{ring} \bar{R}=R / \operatorname{nil}(R)$ is reduced. By Lemma 2.3, $\bar{R}=R / \operatorname{nil}(R)$ satisfies condition $(P)$ and so 3$M$-Armendariz, by [1, Theorem 2.6]. Also, $\alpha \beta \gamma \in \operatorname{nil}(R)[M]$ implies that $\bar{\alpha} \bar{\beta} \bar{\gamma}=\overline{0}$. So $\bar{a}_{i} \bar{b}_{j} \bar{c}_{k}=\overline{0}$, for each $i, j$ and $k$, since $\bar{R}$ is 3 - $M$-Armendariz. Thus $a_{i} b_{j} c_{k} \in \operatorname{nil}(R)$, for each $i, j$ and $k$, and the result follows.

Thus, Theorem 2.10 implies that this class involves $\operatorname{nil}(R) \unlhd R$. Moreover, if we take $M=(\mathbb{N} \cup\{0\},+)$, in Theorem 2.10, it follows that $\operatorname{nil}(R) \unlhd R$ are in fact nil 3-Armendariz [6, Proposition 2.3].

Proposition 2.11. Let $M$ be a u.p.-monoid and $R$ a reduced ring. Then $R$ is nil 3-M-Armendariz.

Proof. Since $R$ is reduced, hence $\operatorname{nil}(R)=0$ is an ideal of $R$. Thus, the result follows from Theorem 2.10.

Corollary 2.12. Let $M$ be a u.p.-monoid and $R$ satisfies condition $(P)$. Then $R$ is nil 3-M-Armendariz.

Proof. Let $M$ be a u.p.-monoid and $R$ satisfies condition $(P)$. Then by $[1$, Theorem 2.6], $R$ is 3 - $M$-Armendariz. Thus, $R$ is nil 3 - $M$-Armendariz.

Corollary 2.13. Let $M$ be a u.p.-monoid and $R$ a semicommutative ring. Then $R$ is nil 3-M-Armendariz.

Proof. Since $R$ is a semicommutative ring, by [11, Lemma 3.1], $\operatorname{nil}(R)$ is an ideal of $R$. Hence the result follows from Theorem 2.10.

Let $(M, \leq)$ be an ordered monoid. If for any $g, g^{\prime}, h \in M, g<g^{\prime}$ implies that $g h<g^{\prime} h$ and $h g<h g^{\prime}$, then $(M, \leq)$ is called a strictly ordered monoid. A monoid $M$ is said to be totally orderable if $(M, \leq)$ is an ordered monoid for some total order $\leq$.

Since each strictly totally ordered monoid is u.p.-monoid, hence we have the following results.

Corollary 2.14. Let $M$ be a strictly totally ordered monoid and $\operatorname{nil}(R)$ an ideal of $R$. Then $R$ is nil 3-M-Armendariz.

Corollary 2.15. Let $M$ be a strictly totally ordered monoid and $R$ a reduced ring. Then $R$ is nil 3-M-Armendariz. 
Clearly $(\mathbb{Z},+)$ is a strictly totally ordered monoid. So a ring $R$ is nil $3-\mathbb{Z}-$ Armendariz, if whenever $\alpha=\Sigma_{i=-n}^{p} a_{i} x^{i}, \beta=\Sigma_{i=-m}^{q} b_{j} x^{j}$ and $\gamma=\Sigma_{k=-r}^{s} c_{k} x^{k} \in$ $R\left[x ; x^{-1}\right]$, satisfy $\alpha \beta \gamma \in \operatorname{nil}(R)\left[x ; x^{-1}\right]$, then $a_{i} b_{j} c_{k} \in \operatorname{nil}(R)$ for each $i, j, k$.

Corollary 2.16. Let $R$ be a ring satisfies condition $(P)$. Then $R$ is nil 3-Z-Z-Armendariz.

Proof. Since $R$ be a ring satisfies condition $(P)$. Then by [1, Corollary 2.8], $R$ is 3 -ZZ-Armendariz, hence $R$ is nil $3-\mathbb{Z}$-Armendariz.

Corollary 2.17. Let $R$ be a semicommutative ring. Then $R$ is nil $3-\mathbb{Z}-$ Armendariz.

Proposition 2.18. Let $M$ be a monoid, $R$ be a nil 3-M-Armendariz ring and $\alpha_{i} \in \operatorname{nil}(R)[M]$, for $1 \leq i \leq n$. If $\alpha_{1} \alpha_{2} \cdots \alpha_{n} \in \operatorname{nil}(R)[M]$, then $a_{1} a_{2} \cdots a_{n} \in \operatorname{nil}(R)$, where $a_{i} \in \operatorname{coef}\left(\alpha_{i}\right)$. In particular, $\operatorname{nil}(R[M]) \subseteq \operatorname{nil}(R)[M]$.

Proof. Let $a_{i}$ be a coefficient of $\alpha_{i}$, for each $i$. We have $\alpha_{1}\left(\alpha_{2} \cdots \alpha_{n}\right) \in$ $\operatorname{nil}(R)[M]$. Thus $a_{1} b \in \operatorname{nil}(R)$, for each $b \in \operatorname{coef}\left(\alpha_{2} \alpha_{3} \cdots \alpha_{n}\right)$, since $R$ is nil 3 - $M$-Armendariz. So $a_{1} \alpha_{2} \alpha_{3} \cdots \alpha_{n} \in \operatorname{nil}(R)[M]$. Hence $\left(a_{1} \alpha_{2}\right)\left(\alpha_{3} \cdots \alpha_{n}\right) \in$ $\operatorname{nil}(R)[M]$. Thus, $a_{1} a_{2} b^{\prime} \in \operatorname{nil}(R)$, for each $b^{\prime} \in \operatorname{coef}\left(\alpha_{3} \alpha_{4} \cdots \alpha_{n}\right)$. By continuing in this way, we have $a_{1} a_{2} \cdots a_{n} \in \operatorname{nil}(R)$ and the proof is complete.

Theorem 2.19. Let $R$ be a ring and $M$ be a monoid. If $\operatorname{nil}(R[M])=$ $\operatorname{nil}(R)[M]$, then $R$ is nil 3-M-Armendariz.

Proof. Suppose $\alpha=\sum_{i=1}^{n} a_{i} g_{i}, \beta=\sum_{j=1}^{m} b_{j} h_{j}$ and $\gamma=\sum_{k=1}^{r} c_{k} l_{k}$ be elements of $R[M]$ be such that $\alpha \beta \gamma \in \operatorname{nil}(R)[M]=\operatorname{nil}(R[M])$. So there exists a positive integer $s$ such that $(\alpha \beta \gamma)^{s}=0$. Therefore, we have $\left(a_{i} b_{j} c_{k}\right)^{s}=$ $\left(a_{i} b_{j} c_{k}\right) \cdots\left(a_{i} b_{j} c_{k}\right)=0$, by Proposition 2.18 and thus $a_{i} b_{j} c_{k} \in \operatorname{nil}(R)$, for each $i, j$ and $k$. Hence $R$ is a nil $3-M$-Armendariz ring.

Observe that if $\operatorname{nil}(R)$ is an ideal, then by [6, Proposition 2.6], $R$ is nil 3 -Armendariz. More generally we obtain the following.

Proposition 2.20. Let $M$ be a monoid and $I \subseteq R$ be a nil ideal of $R$. Then $R$ is nil 3-M-Armendariz if and only if so is $R / I$.

Proof. Since $I \subseteq \operatorname{nil}(R)$, we have $\operatorname{nil}(R / I)=\operatorname{nil}(R) / I$. So $\alpha \beta \gamma \in \operatorname{nil}(R)[M]$ if and only if $\bar{\alpha} \bar{\beta} \bar{\gamma} \in \operatorname{nil}(R / I)[M]$. Also, abc $\in \operatorname{nil}(R)$ if and only if $\bar{a} \bar{b} \bar{c} \in$ $\operatorname{nil}(R / I)$. Therefore $R$ is nil $3-M$-Amendariz if and only if $R / I$ is nil $3-M$ Armendariz. 
Taking $M=(\mathbb{N} \cup\{0\},+)$, in Proposition 2.20, it follows that for any nil ideal $I \subseteq R$, we have $R$ is nil 3-Armendariz if and only if so is $R / I$ [6, Proposition $2.6]$.

Recall that an element $u$ of a ring $R$ is right regular if $u r=0$ implies $r=0$ for $r \in R$. Similarly, left regular elements can be defined. An element is regular if it is both left and right regular (and hence not a zero divisor).

Proposition 2.21. Let $R$ be a ring and $\Delta$ be a multiplicative monoid in $R$ consisting of central regular elements. Then $R$ is nil 3-M-Armendariz if and only if so is $\Delta^{-1} R$.

Proof. Let $R$ be nil 3-M-Armendariz ring, and $S=\Delta^{-1} R$. Put $\alpha \beta \gamma=0$, where $\alpha=\sum_{i=1}^{n} a_{i} g_{i}, \beta=\sum_{j=1}^{m} b_{j} h_{j}$ and $\gamma=\sum_{k=1}^{r} c_{k} l_{k} \in S[M]$. We may assume that $a_{i}=\varepsilon_{i} u^{-1}, b_{j}=\eta_{j} v^{-1}$ and $c_{k}=\mu_{k} w^{-1}$ with $\varepsilon_{i}, \eta_{j}, \mu_{k}$ are in $R$ for all $i, j$ and $k$, and $u, v, w \in \Delta$. We will show that $a_{i} b_{j} c_{k} \in \operatorname{nil}(S)$. Now we have

$$
\begin{aligned}
\operatorname{nil}(S)[M] & \ni \alpha \beta \gamma \\
& =\sum_{i=1}^{n} \sum_{j=1}^{m} \sum_{k=1}^{r} a_{i} b_{j} c_{k} g_{i} h_{j} l_{k} \\
& =\sum_{i=1}^{n} \sum_{j=1}^{m} \sum_{k=1}^{r} \varepsilon_{i} \eta_{j} \mu_{k} u^{-1} v^{-1} w^{-1} g_{i} h_{j} l_{k} \\
& =\left(\sum_{i=1}^{n} \sum_{j=1}^{m} \sum_{k=1}^{r} \varepsilon_{i} \eta_{j} \mu_{k} g_{i} h_{j} l_{k}\right)(u v w)^{-1} .
\end{aligned}
$$

Hence

$$
\sum_{i=1}^{n} \sum_{j=1}^{m} \sum_{k=1}^{r} \varepsilon_{i} \eta_{j} \mu_{k} g_{i} h_{j} l_{k} \in \operatorname{nil}(R)[M] .
$$

Since $R$ is nil 3 - $M$-Armendariz, $\varepsilon_{i} \eta_{j} \mu_{k} \in \operatorname{nil}(R)$ for all $i, j$ and $k$ and so

$$
a_{i} b_{j} c_{k}=\varepsilon_{i} u^{-1} \eta_{j} v^{-1} \mu_{k} w^{-1}=\varepsilon_{i} \eta_{j} \mu_{k}(u v w)^{-1} \in \operatorname{nil}(S),
$$

for all $i, j, k$. Thus, $S$ is nil 3 - $M$-Armendariz. The converse it is obvious that subring of nil 3 - $M$-Armendariz is nil $3-M$-Armendariz.

Proposition 2.22. Let $M$ be a monoid, $R$ be a ring and $e$ an idempotent of $R$. If $e$ is central in $R$, then the following statements are equivalent:

1. $R$ is nil $3-M-A r m e n d a r i z ;$

2. $e R$ and $(1-e) R$ are nil 3 - $M$-Armendariz.

Proof. We only need to prove $(2) \Rightarrow(1)$. Let $\alpha=\sum_{i=1}^{n} a_{i} g_{i}, \beta=\sum_{j=1}^{m} b_{j} h_{j}$ and $\gamma=\sum_{k=1}^{r} c_{k} l_{k}$ be elements in $R[M]$ be such that $\alpha \beta \gamma \in \operatorname{nil}(R)[M]$. Let $\alpha_{1}=\Sigma_{i=1}^{n}\left(e a_{i}\right) g_{i}, \alpha_{2}=\Sigma_{i=1}^{n}(1-e) a_{i} g_{i}, \beta_{1}=\Sigma_{j=1}^{m}\left(e b_{j}\right) h_{j}, \beta_{2}=\Sigma_{j=1}^{m}(1-e) b_{j} h_{j}$ and $\gamma_{1}=\Sigma_{k=1}^{r}\left(e c_{k}\right) l_{k}, \gamma_{2}=\Sigma_{k=1}^{r}(1-e) c_{k} l_{k}$. So $\alpha_{1} \beta_{1} \gamma_{1} \in \operatorname{nil}(e R)[M]$ and 
$\alpha_{2} \beta_{2} \gamma_{2} \in \operatorname{nil}((1-e) R)[M]$. Thus $e a_{i} b_{j} c_{k} \in \operatorname{nil}(e R)$ and $(1-e) a_{i} b_{j} c_{k} \in \operatorname{nil}((1-$ $e) R$ ), since $e R$ and $(1-e) R$ are nil 3 - $M$-Armendariz. Therefore $e\left(a_{i} b_{j} c_{k}\right)^{u_{i j k}}$ and $(1-e)\left(a_{i} b_{j} c_{k}\right)^{v_{i j k}}=0$, for some positive integer $u_{i j k}$ and $v_{i j k}$. If we take $s=\max \left\{u_{i j k}, v_{i j k} \mid 1 \leq i \leq n, 1 \leq j \leq m, 1 \leq k \leq r\right\}$, then we have $e\left(a_{i} b_{j} c_{k}\right)^{s}=$ $(1-e)\left(a_{i} b_{j} c_{k}\right)^{s}=0$, for each $i, j, k$. Thus, $\left(a_{i} b_{j} c_{k}\right)^{s}=0$ and so $a_{i} b_{j} c_{k} \in \operatorname{nil}(R)$, for each $i, j, k$. This implies that $R$ is nil 3 -M-Armendariz and the proof is complete.

Proposition 2.23. Let $M$ be a monoid. If $R$ is a semicommutative ring which is also nil 3-M-Armendariz, then we have $\operatorname{nil}(R[M])=\operatorname{nil}(R)[M]$.

Proof. Since $R$ is nil 3-M-Armendariz, we have $\operatorname{nil}(R[M]) \subseteq \operatorname{nil}(R)[M]$, by Proposition 2.18. Now, let $\alpha=a_{1} g_{1}+\cdots+a_{n} g_{n} \in \operatorname{nil}(R)[M]$, and let $k>1$ such that $a_{i}^{k}=0$ for all $i=1, \cdots, n$. We claim that $\alpha^{n k}=0$. The coefficients of $\alpha^{n k}$ can be written as sums of monomials of length $n k$ in the $a_{i}$ 's. Consider one of these monomials, $a_{i_{1}} a_{i_{2}} \cdots a_{i_{n k}}$ where $1 \leq i_{j} \leq n$. It must contain at least $k$ occurrences of some $a_{j 0}$ for some $1 \leq j_{0} \leq n$. Since $a_{j_{0}}^{k}=0$ and $R$ is semicommutative, we have $a_{i_{1}} a_{i_{2}} \cdots a_{i_{n k}}=0$. Therefore, we have proved that all the monomials appearing in the coefficients of $\alpha^{n k}$ are 0 . Hence $\operatorname{nil}(R)[M] \subseteq \operatorname{nil}(R[M])$, and so $\operatorname{nil}(R[M])=\operatorname{nil}(R)[M]$.

Zhongkui [10, Proposition 2.1], it was shown that if $R$ is a reduced and $M$-Armendariz ring, then $R[M]$ is $N$-Armendariz, where $M$ is a monoid and $N$ a u.p.-monoid. Also Elshokry and et al. [1, Proposition 3.1], it was shown that if $R$ satisfies condition $(P)$, and is $3-M$-Armendariz, then $R[M]$ is $3-N$ Armendariz. For nil 3-M-Armendariz, we have the following results.

Proposition 2.24. Let $M$ be a monoid and $N$ a u.p.-monoid. If $R$ is a semicommutative ring which is also nil $3-M$-Armendariz, then $R[M]$ is nil 3 - $N$-Armendariz.

Proof. By Proposition 2.23, $\operatorname{nil}(R[M])=\operatorname{nil}(R)[M]$ is an ideal of $R[M]$. Since $N$ is a u.p.-monoid, hence by Theorem 2.10, $R[M]$ is nil 3 - $N$-Armendariz.

Proposition 2.25. Let $M$ be a monoid and $N$ a u.p.-monoid. If $R$ is a semicommutative ring which is also nil 3-M-Armendariz, then $R[N]$ is nil 3-M-Armendariz. 
Proof. It is easy to see that there exists an isomorphism of rings $R[N][M] \cong$ $R[M][N]$ defined by

$$
\sum_{p}\left(\sum_{i} a_{i p} n_{i}\right) m_{p} \rightarrow \sum_{i}\left(\sum_{p} a_{i p} m_{p}\right) n_{i}
$$

Now suppose that $\alpha_{i}, \beta_{j}, \gamma_{k} \in R[N]$ are such that $\left(\sum_{i} \alpha_{i} g_{i}\right)\left(\sum_{j} \beta_{j} h_{j}\right)\left(\sum_{k} \gamma_{k} l_{k}\right) \in$ $\operatorname{nil}(R[N])[M]$, where $g_{i}, h_{j}, l_{k} \in M$. We will show that $\alpha_{i} \beta_{j} \gamma_{k} \in \operatorname{nil}(R[N])$ for all $i, j$ and $k$. Assume that $\alpha_{i}=\sum_{p} a_{i p} n_{p}, \beta_{j}=\sum_{q} b_{j q} n_{q}^{\prime}$ and $\gamma_{k}=\sum_{s} c_{k s} n_{s}^{\prime \prime}$, where $n_{p}, n_{q}^{\prime}, n_{s}^{\prime \prime} \in N$ for all $p, q$ and $s$. Then

$$
\left(\sum_{i}\left(\sum_{p} a_{i p} n_{p}\right) g_{i}\right)\left(\sum_{j}\left(\sum_{q} b_{j q} n_{q}^{\prime}\right) h_{j}\right)\left(\sum_{k}\left(\sum_{s} c_{k s} n_{s}^{\prime \prime}\right) l_{k}\right) \in \operatorname{nil}(R[N])[M]
$$

Thus, in $R[M][N]$ we have

$$
\left(\sum_{p}\left(\sum_{i} a_{i p} g_{i}\right) n_{p}\right)\left(\sum_{q}\left(\sum_{j} b_{j q} h_{j}\right) n_{q}^{\prime}\right)\left(\sum_{s}\left(\sum_{k} c_{k s} l_{k}\right) n_{s}^{\prime \prime}\right) \in \operatorname{nil}(R[M])[N] .
$$

By Proposition 2.24, $R[M]$ is nil 3-N-Armendariz,

$$
\left(\sum_{i} a_{i p} g_{i}\right)\left(\sum_{j} b_{j q} h_{j}\right)\left(\sum_{k} c_{k s} l_{k}\right) \in \operatorname{nil}(R[M])
$$

for all $p, q$ and $s$. Since $R$ is nil 3 -M-Armendariz, $a_{i p} b_{j q} c_{k s} \in \operatorname{nil}(R)$ for all $i, j, k, p, q, s$. Hence $\alpha_{i} \beta_{j} \gamma_{k} \in \operatorname{nil}(R[N])$. This means that $R[N]$ is nil $3-M-$ Armendariz.

Corollary 2.26. Let $M$ be a monoid and $R$ a semicommutative ring. If $R$ is nil 3-M-Armendariz, then $R[x]$ and $R\left[x ; x^{-1}\right]$ are nil $3-M$-Armendariz.

Proof. Since $R[x] \cong R[\mathbb{N} \cup\{0\}]$ and $R\left[x ; x^{-1}\right] \cong R[\mathbb{Z}]$, the result follows from Proposition 2.25.

Theorem 2.27. Let $M$ be a monoid and $N$ a u.p.-monoid. If $R$ a semicommutative ring which is also nil 3-M-Armendariz, then $R$ is nil $3-(M \times N)$ Armendariz.

Proof. By [1, Theoerem 3.3], $R[M \times N] \cong R[M][N]$, and by Proposition $2.23, \operatorname{nil}(R[M])=\operatorname{nil}(R)[M]$. Now the assertion follows from Proposition 2.24. 
Let $M_{i}, i \in I$, be monoids. Denote $\coprod_{i \in I} M_{i}=\left\{\left(g_{i}\right)_{i \in I} \mid\right.$ there exist only finite $i$ 's such that $g_{i} \neq e_{i}$, the identity of $\left.M_{i}\right\}$. Then $\coprod_{i \in I} M_{i}$ is a monoid with the operation $\left(g_{i}\right)_{i \in I}\left(g_{i}^{\prime}\right)_{i \in I}=\left(g_{i} g_{i}^{\prime}\right)_{i \in I}$.

Corollary 2.28. Let $M_{i}, i \in I$ be u.p.-monoids and $R$ a semicommutative ring. If $R$ is nil $3-M_{i_{0}}$-Armendariz for some $i_{0} \in I$, then $R$ is nil $3-\coprod_{i \in I} M_{i^{-}}$ Armendariz.

Proof. Let $\alpha=\Sigma_{i} a_{i} g_{i}, \beta=\Sigma_{j} b_{j} h_{j}, \gamma=\Sigma_{k} c_{k} l_{k} \in R\left[\coprod_{i \in I} M_{i}\right]$ such that $\alpha \beta \gamma \in \operatorname{nil}\left(R\left[\amalg_{i \in I} M_{i}\right]\right)$. Then $\alpha, \beta, \gamma \in R\left[M_{1} \times M_{2} \times \cdots \times M_{n}\right]$, for some finite subset $\left\{M_{1}, M_{2}, \cdots, M_{n}\right\} \subseteq\left\{M_{i} \mid i \in I\right\}$. Thus $\alpha, \beta, \gamma \in R\left[M_{i_{0}} \times M_{1} \times\right.$ $\left.M_{2} \times \cdots \times M_{n}\right]$. The ring $R$, by Theorem 2.27 and by induction, is nil 3$\left(M_{i_{0}} \times M_{1} \times M_{2} \times \cdots \times M_{n}\right)$-Armendariz, so $a_{i} b_{j} c_{k} \in \operatorname{nil}(R)$ for all $i, j$ and $k$. Hence $R$ is nil $3-\coprod_{i \in I} M_{i}$-Armendariz.

\section{Acknowledgments}

We would like to thank Professor Liu Zhongkui for his valuable comments and we would like to thank the managements of University of Khartoum and Northwest Normal University. Also the authors thank the referee for a very careful reading of the paper.

\section{References}

[1] A. Elskokry, E. Ali, L. Zhongkui, On the extension of Armendariz rings relative to a monoid, (Accepted).

[2] C. Huh, Y. Lee and A. Smoktunowicz, Armendariz rings and semicommutative rings, Comm. Algebra, 30, No 2 (2002), 751-761.

[3] C. Y. Hong, N.K. Kim and T.K. Kwak, On Skew Armendariz rings, Comm. Algebra, 31, N0 1 (2003), 103-122.

[4] D. D. Anderson, V. Camillo, Armendariz rings and Gaussian rings, Comm. Algebra, 26, No 7 (1998), 2265-2272.

[5] D. S. Passman, The Algebraic Structure of Group Rings, John Wiley, New York, (1977). 
[6] E. Ali, A. Elshokry, L. Zhongkui, Nil 3-Armendariz rings, Advances. Pure. Math, 3, No 9 (2013), 703-708.

[7] E. Hashemi, Nil-Armendariz rings relative to a monoid, Mediter. J. Math, 10, No 1 (2013), 111-121.

[8] E. P. Armendariz, A note on extensions of Baer and p.p.-rings, J. Austral. Math. Soc., 18 (1974), 470-473.

[9] G. F. Birkenmeier, J.K. Park, Triangular matrix representations of ring extensions, J. Algebra, 265 (2003), 457-477.

[10] L. Zhongkui, Armendariz rings relative to a monoid, Comm. Algebra, 33, No 3 (2005), 649-661.

[11] L. ZhongKui, R. Y. Zhao, On weak Armendariz rings, Comm. Algebra, 34, No 7 (2006), 2607-2616.

[12] M. B. Rege, S. Chhawchharia, Armendariz rings, Proc. Japan Acad. Ser. A math. Sci., 73 (1997), 14-17.

[13] M. Habibi, A. Moussavi, Nilpotent elements and nil Armendariz property of monoid rings, J. Algebra, App, 11, No 4 (2012), 1250080-1-1250080-14.

[14] M. J. Nikmehr, F. Fatahi and H. Amraei, Nil-Armendariz rings with Applications to a monoid, World App. Sci. J, 13, No 12 (2011), 2509-2514.

[15] N. K. Kim, Y. Lee, Armendariz rings and reduced rings, J. Algebra, 223 (2000), 477-488.

[16] R. Antoine, Nilpotent elements and Armendariz rings, J. Algebra 319, (2008), 3128-3140.

[17] T.K. Lee, T.L. Wong, On Armendariz rings, Houston J. Math. 29, No 3 (2003), 583-593.

[18] Wu. Hui-feng, Extensions of Reduced Rings, J. Hangzhou Normal Uni, 10, No 5 (2011), 407-410.

[19] Wu Hui-feng, On Weak 3-Armendariz rings, J. Hangzhou Normal Uni, 11, No 3 (2012), 241-244.

[20] Y. Suiyi, On the extension of Armendariz rings, [D]. Lanzhou University, (2008). 
[21] Z. Cuiping, C. Jianlong, Weak $M$-Armendariz rings, J. Southeast Uni, 25, No 1 (2009) 142-146. 
